\title{
A Practical Mild, One-Pot, Regiospecific Synthesis of 2, 3-Disubstituted Indoles via Consecutive Sonogashira and Cacchi Reactions
}

Bruce Z. Lu, ${ }^{*}$ Wenyi Zhao, Han-Xun Wei, Marine Dufour, Vittorio Farina, ${ }^{\dagger}$ and Chris H. Senanayake ${ }^{\dagger}$

Department of Chemical Development, Boehringer-Ingelheim Pharmaceuticals, Inc., 800 East Leigh Street, Suite 205, Richmond, VA 23219;

$\dagger 900$ Ridgebury Rd, Ridgefield, CT 06877.

\section{Supporting Information}

General: All reactions were carried out in oven-dried glassware sealed with rubber septa under an inert atmosphere of dry argon. All commercial materials were used without further purification. Analytical data were obtained from high performance liquid chromatography (HPLC) on Agilent 1100 Series equipped with a UV detector and Eclipse XDB-C8 4.6 x $150 \mathrm{~mm} 5 \mu \mathrm{m}$ column. A thin layer chromatography (TLC) was visualized by ultraviolet light $(254 \mathrm{~nm})$. Chemical yields refer to pure isolated substances. Purification of products was accomplished by column chromatography packed with silica gel. ${ }^{1} \mathrm{H}$ and ${ }^{13} \mathrm{C}$ NMR were recorded using a Bruker 400 . Chemical shifts are reported in ppm. The following abbreviations were used to designate chemical shift multiplicities: $s$ = singlet, $d=$ doublet, $t=$ triplet, $q=$ quartet, $h=$ heptet, $m=$ multiplet, $b r=$ broad. LCMS was recorded on Agilent MSD spectrometer with VL API-ES positive scan. Elemental analyses were obtained from Quantitative Technologies, Inc.

\section{General Procedure for One-pot Domino Reaction Condition (Method A):}

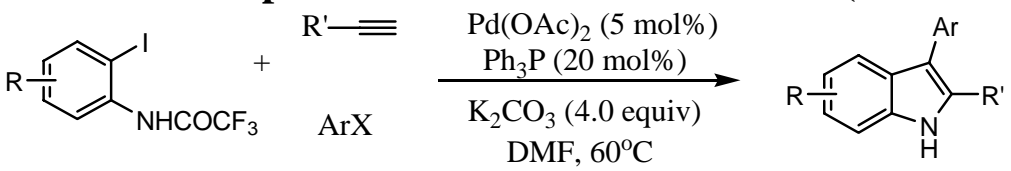

A $10 \mathrm{~mL}, 3$-neck flask, equipped with a magnetic-stirring bar and thermocouple, and Argon inlet, were charged 2-trifluoroacetylamido-iodobenzene ( $0.5 \mathrm{~g}, 1.54 \mathrm{mmol}, 1.0$ equiv), $\mathrm{Pd}(\mathrm{OAc})_{2}(17.32 \mathrm{mg}, 0.08 \mathrm{mmol}, 0.05$ equiv), triphenylphosphine $(80.94 \mathrm{mg}$, $0.154 \mathrm{mmol}, 0.2$ equiv), and potassium carbonate $(0.851 \mathrm{~g}, 6.16 \mathrm{mmol}, 4$ equiv), followed by addition of $5 \mathrm{~mL}$ of anhydrous DMF. With stirring at room temperature, were added into the reaction mixture aryl acetylene $(1.85 \mathrm{mmol}, 1.2$ equiv) and aryl bromide ( $1.85 \mathrm{mmol}, 1.2$ equiv). The reaction was heated then at $60^{\circ} \mathrm{C}$ until the reaction was complete monitored by HPLC. The mixture was quenched with water, and the aqueous solution was extracted with AcOEt three times. The organic solution was washed with and brine, dried over $\mathrm{Na}_{2} \mathrm{SO}_{4}$. The product was purified by column chromatography.

2,3-Diphenyl-1H-indole (3) (Fennell, R. C. G.; Plant, S. G. P. J. Chem. Soc. 1932, 28722874.) (Table 2, entry 1): $91 \%$ yield*, off-white solid, mp $108-110^{\circ} \mathrm{C}$. ${ }^{1} \mathrm{H}$ NMR (400 $\mathrm{MHz}, \mathrm{DMSO}) \delta$ (ppm): 11.55 (s, 1H), 7.46-6.90 (m, 14H); ${ }^{13} \mathrm{C}$ NMR (400 MHz, $\mathrm{CDCl}_{3}$ ) $\delta$ (ppm): 135.89, 135.05, 134.08, 132.71, 130.16, 128.76, 128.70, 128.52, 128.17, 127.70, 126.23, 122.71, 120.44, 119.71, 115.07, 110.87. LC-MSD (API-ES, positive) $\mathrm{m} / \mathrm{z}=270$ $\left(\mathrm{M}+\mathrm{H}^{+}\right)$. 
Methyl 6-(2,3-diphenyl-indolyl)carboxylate (8) (Coldham, M. W. G.; Lewis, J. W.; Plant, S. G. P. J. Chem. Soc. 1954, 4528-4530.) (Table 2, enry 5): $86 \%$ \%, pale brown solid, mp 234-236 ${ }^{\circ} \mathrm{C} .{ }^{1} \mathrm{H}$ NMR $\left(400 \mathrm{MHz}, \mathrm{CDCl}_{3}\right) \delta(\mathrm{ppm}): 8.54(\mathrm{~s}, 1 \mathrm{H}), 8.20(\mathrm{~d}, \mathrm{~J}=0.8$ $\mathrm{Hz}, 1 \mathrm{H}), 7.85-7.68(\mathrm{~m}, 2 \mathrm{H}), 7.47-7.34(\mathrm{~m}, 10 \mathrm{H}), 3.95(\mathrm{~s}, 3 \mathrm{H}) ;{ }^{13} \mathrm{C}$ NMR $(400 \mathrm{MHz}$, $\left.\mathrm{CDCl}_{3}\right) \delta$ (ppm): 168.07, 137.43, 135.17, 134.38, 132.28, 132.05, 130.12, 128.83, 128.66, 128.33, 128.30, 126.60, 124.19, 121.53, 119.24, 115.46, 113.27, 52.03; LC-MSD (APIES, positive $) \mathrm{m} / \mathrm{z}=328\left(\mathrm{M}+\mathrm{H}^{+}\right)$.

Methyl 6-[2-(p-methylphenyl)-3-phenylindolyl]carboxylate (7) (Table 2, entry 4): 82 $\%$, pale brown solid, mp 227-229 ${ }^{\circ} \mathrm{C} .{ }^{1} \mathrm{H}$ NMR (400 MHz, $\left.\mathrm{CDCl}_{3}\right) \delta(\mathrm{ppm}): 8.56(\mathrm{~s}, 1 \mathrm{H})$, $8.20(\mathrm{t}, \mathrm{J}=0.6 \mathrm{~Hz}, 1 \mathrm{H}), 7.83-7.81(\mathrm{~m}, 1 \mathrm{H}), 7.66(\mathrm{~d}, \mathrm{~J}=8.4 \mathrm{~Hz}, 1 \mathrm{H}), 7.43-7.28(\mathrm{~m}, 7 \mathrm{H})$, $7.15(\mathrm{~d}, \mathrm{~J}=8.0 \mathrm{~Hz}, 2 \mathrm{H}), 3.93(\mathrm{~s}, 3 \mathrm{H}), 2.36(\mathrm{~s}, 3 \mathrm{H}) ;{ }^{13} \mathrm{C} \mathrm{NMR}\left(400 \mathrm{MHz}, \mathrm{CDCl}_{3}\right)$ $\delta$ (ppm): 168.15, 138.34, 137.66, 135.10, 134.55, 132.35, 130.11, 129.53, 129.13, 128.63, 128.14, 126.51, 123.93, 121.44, 119.06, 115.00, 113.24, 52.01, 21.31. LC-MSD (API-ES, positive $) \mathrm{m} / \mathrm{z}=342\left(\mathrm{M}+\mathrm{H}^{+}\right)$. Elemental analysis (Calcd, \%): C, 80.92; H, 5.61; N, 4.10; found: C, 80.18, H, 5.64, N, 3.96.

2,3-Diphenyl-5-cyano-1H-indole (11) (Table 2, entry 8): $91 \% *$, as pale brown solid, decomposed at $157^{\circ} \mathrm{C} .{ }^{1} \mathrm{H}$ NMR (400 MHz, DMSO-d 6 ) $\delta(\mathrm{ppm}): 12.20(\mathrm{~s}, 1 \mathrm{H}), 7.89$ (s, $1 \mathrm{H})$, 7.62-7.30 (m, 12H); ${ }^{13} \mathrm{C}$ NMR (400 MHz, DMSO-d 6 ) $\delta(\mathrm{ppm}): 137.70,136.50$, 133.72 , 131.33, 129.66, 128.76, 128.57, 128.26, 128.19, 127.64, 126.62, 124.63, 124.01, $120.43,113.79,112.68,101.77$.

2-Phenyl-3-(p-methoxyphenyl)-1H-indole (5) (Table 2, entry 2): 60\%*, as pale brown solid, mp 185-187.5 ${ }^{\circ} \mathrm{C} .{ }^{1} \mathrm{H}$ NMR $\left(400 \mathrm{MHz}, \mathrm{CDCl}_{3}\right) \delta(\mathrm{ppm}): 8.21(\mathrm{~s}, 1 \mathrm{H}), 7.65(\mathrm{~d}, \mathrm{~J}=$ $8.0 \mathrm{~Hz}, 1 \mathrm{H}), 7.44-6.92(\mathrm{~m}, 12 \mathrm{H}), 3.85(\mathrm{~s}, 3 \mathrm{H}) .{ }^{13} \mathrm{C} \mathrm{NMR}\left(400 \mathrm{MHz}, \mathrm{CDCl}_{3}\right) \delta(\mathrm{ppm})$ : $158.17,135.86,133.70,132.84,131.19,128.99,128.68,128.04,127.56,127.34,122.64$, 121.02, 119.71, 114.73, 114.05, 110.83, 55.24. Elemental analysis (Calcd, \%): C, 84.25; H, 5.72; N, 4.68; found: C, 83.80, H, 5.62, N, 4.54.

2-Phenyl-3-(p-methoxyphenyl)-5-cyano-1H-indole (12) (Table 2, entry 9): $78 \% *$, as pale brown solid, mp 209-212 ${ }^{\circ} \mathrm{C} .{ }^{1} \mathrm{H}$ NMR (400 $\left.\mathrm{MHz}, \mathrm{CDCl}_{3}\right) \delta(\mathrm{ppm}): 8.51(\mathrm{~s}, 1 \mathrm{H})$, $8.05(\mathrm{~s}, 1 \mathrm{H}), 7.97-7.29(\mathrm{~m}, 9 \mathrm{H}), 6.96(\mathrm{~d}, \mathrm{~J}=8.4 \mathrm{~Hz}, 2 \mathrm{H}), 3.90(\mathrm{~s}, 3 \mathrm{H}) .{ }^{13} \mathrm{C}$ NMR $(400$ $\left.\mathrm{MHz}, \mathrm{CDCl}_{3}\right) \delta(\mathrm{ppm}): 158.68,137.21,135.09,131.56,131.06,128.98,128.93,128.39$, $128.06,125.80,125.50,125.89,120.98,115.08,114.39,111.67,103.89,55.31$.

\section{General Procedure for Sequential Addition Reaction Condition (Method B):}

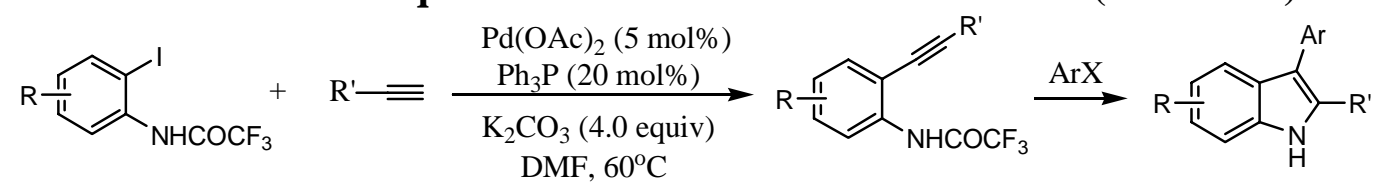

A $10 \mathrm{~mL}, 3$-neck flask, equipped with a magnetic-stirring bar and thermocouple, and Argon inlet, were charged 2-trifluoroacetylamido-iodobenzne (0.5 g, $1.54 \mathrm{mmol}, 1.0$ equiv), $\mathrm{Pd}(\mathrm{OAc})_{2}(17.32 \mathrm{mg}, 0.08 \mathrm{mmol}, 0.05$ equiv), triphenylphosphine $(80.94 \mathrm{mg}$, 
$0.154 \mathrm{mmol}, 0.2$ equiv), and potassium carbonate $(0.851 \mathrm{~g}, 6.16 \mathrm{mmol}, 4$ equiv), followed by addition of $5 \mathrm{~mL}$ of anhydrous DMF. With stirring at room temperature, was added into the reaction mixture aryl acetylene $(1.85 \mathrm{mmol}, 1.2$ equiv. The reaction was heated then at $60^{\circ} \mathrm{C}$ until the Sonogashira reaction was complete judged by monitoring the disappearance of the iodoamide with HPLC. After the completion of the Sonogashira reaction, it was added aryl bromide ( $1.85 \mathrm{mmol}, 1.2$ equiv). The mixture was quenched with water upon the Sonogashira product disappeared, and the aqueous solution was extracted with AcOEt three times. The organic solution was washed with and brine, dried over $\mathrm{Na}_{2} \mathrm{SO}_{4}$. The product was purified by column chromatography.

2-Phenyl-3-( $\boldsymbol{p}$-methoxycarbonylphenyl)-1H-indole (6) (Cowper, R. M.; Stevens, T. S. J. Chem. Soc. 1947, 1041-1044.) (Table 2, entry 3): $85 \%$ yield, pale brown solid, mp 155-157 ${ }^{\circ} \mathrm{C} .{ }^{1} \mathrm{H}$ NMR $\left(400 \mathrm{MHz}, \mathrm{CDCl}_{3}\right) \delta(\mathrm{ppm}): 8.34(\mathrm{~s}, 1 \mathrm{H}), 8.03(\mathrm{~d}, \mathrm{~J}=8.4 \mathrm{~Hz}, 2 \mathrm{H})$, $7.70(\mathrm{~d}, \mathrm{~J}=8.0 \mathrm{~Hz}, 1 \mathrm{H}), 7.53-7.21(\mathrm{~m}, 10 \mathrm{H}), 3.93(\mathrm{~s}, 3 \mathrm{H}) ;{ }^{13} \mathrm{C} \mathrm{NMR}\left(400 \mathrm{MHz}, \mathrm{CDCl}_{3}\right)$ $\delta$ (ppm): 167.27, 140.33, 135.98, 135.05, 132.32, 129.90, 128.88, 128.38, 128.26, 128.14, 127.71, 126.85, 123.00, 120.85, 119.46, 114.02, 111.08, 52.05. LC-MSD (API-ES, positive) $\mathrm{m} / \mathrm{z}=328\left(\mathrm{M}+\mathrm{H}^{+}\right)$.

Methyl 6-[2-phenyl-3-(o-nitrophenyl)indolyl]carboxylate (9) (Table 2, entry 6) : $94 \%$, pale brown solid, mp 233-236 ${ }^{\circ} \mathrm{C} .{ }^{1} \mathrm{H}$ NMR $\left(400 \mathrm{MHz}, \mathrm{CDCl}_{3}\right) \delta(\mathrm{ppm}): 8.65(\mathrm{~s}, 1 \mathrm{H})$, $8.20(\mathrm{t}, \mathrm{j}=0.6 \mathrm{~Hz}, 1 \mathrm{H}), 7.98-7.95(\mathrm{~m}, 1 \mathrm{H}), 7.82-7.80(\mathrm{~m}, 1 \mathrm{H}), 7.62-7.44(\mathrm{~m}, 3 \mathrm{H}), 7.39-$ $7.37(\mathrm{~m}, 6 \mathrm{H}), 3.95(\mathrm{~s}, 3 \mathrm{H}) .{ }^{13} \mathrm{C}$ NMR $\left(400 \mathrm{MHz}, \mathrm{CDCl}_{3}\right) \delta(\mathrm{ppm}): 167.87,150.44$, $138.35,135.06,133.95,132.74,131.89,131.16,129.36,129.06,128.74,128.17,127.88$, 124.69, 124.58, 121.95, 118.36, 113.55, 110.55, 52.06. LC-MSD (API-ES, positive) m/z $=373\left(\mathrm{M}+\mathrm{H}^{+}\right)$. Elemental analysis $($Calcd, \%): $\mathrm{C}, 70.96 ; \mathrm{H}, 4.33 ; \mathrm{N}, 7.52$; found: $\mathrm{C}$, $70.75 ; \mathrm{H}, 4.38 ; \mathrm{N}, 6.83$.

2-Phenyl-3-( $\boldsymbol{p}$-nitrophenyl)-1H -indole (10) (Table 2, entry 7): $98 \%$ *, pale brown solid, mp 219-222 ${ }^{\circ} \mathrm{C} .{ }^{1} \mathrm{H}$ NMR $(400 \mathrm{MHz}$, DMSO-d 6 ) $\delta(\mathrm{ppm}): 11.88(\mathrm{~s}, 1 \mathrm{H}), 8.23(\mathrm{~d}, \mathrm{~J}=8.8$ $\mathrm{Hz}, 2 \mathrm{H}), 7.68-7.37(\mathrm{~m}, 10 \mathrm{H}), 7.24-7.11(\mathrm{~m}, 2 \mathrm{H}) .{ }^{13} \mathrm{C}$ NMR (400 MHz, DMSO-d 6 ) $\delta$ (ppm): 145.06, 143.02, 136.26, 136.11, 131.76, 130.21, 128.81, 128.70, 128.27, 127.05, $123.89,122.49,120.48,118.30,111.85,111.14$.

2-Phenyl-3-(o-nitrophenyl)-5-cyano-1H-indole (13) (Table 2, entry 10): $80 \% *$, pale brown solid, mp $268-270^{\circ} \mathrm{C} .{ }^{1} \mathrm{H}$ NMR $\left(400 \mathrm{MHz}, \mathrm{DMSO}^{-} \mathrm{d}_{6}\right) \delta(\mathrm{ppm}): 12.35(\mathrm{~s}, 1 \mathrm{H})$, $8.05(\mathrm{~d}, \mathrm{~J}=8 \mathrm{~Hz}, 1 \mathrm{H}), 7.78-7.37(\mathrm{~m}, 11 \mathrm{H}) ;{ }^{13} \mathrm{C}$ NMR $(400 \mathrm{MHz}$, DMSO-d 6 ) $\delta(\mathrm{ppm})$ : $150.01,137.65,137.35,133.67,133.33,130.51,128.93$, 128.76, 128.45, 127.95, 127.69, $127.56,124.85,124.51,123.34,120.18,112.86,109.03,101.98$. Elemental analysis (Calcd, \%): C, 74.33; H, 3.86; N, 12.38; Found: C, 73.82; H, 3.70; N, 12.00.

* Yields were determined by solution assay. 


\section{2,3-Diphenyl-1H-indole (3) $\left({ }^{1} \mathrm{H}\right.$ NMR) (Table 2, entry 1)}

மำ

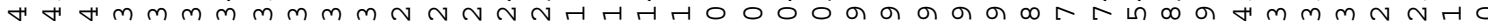

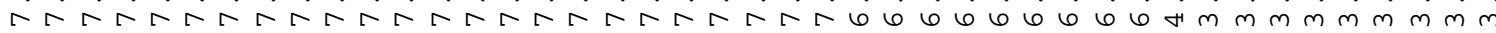

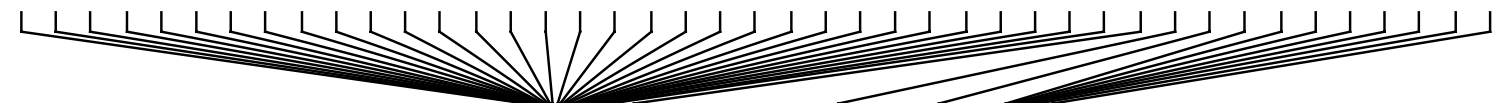

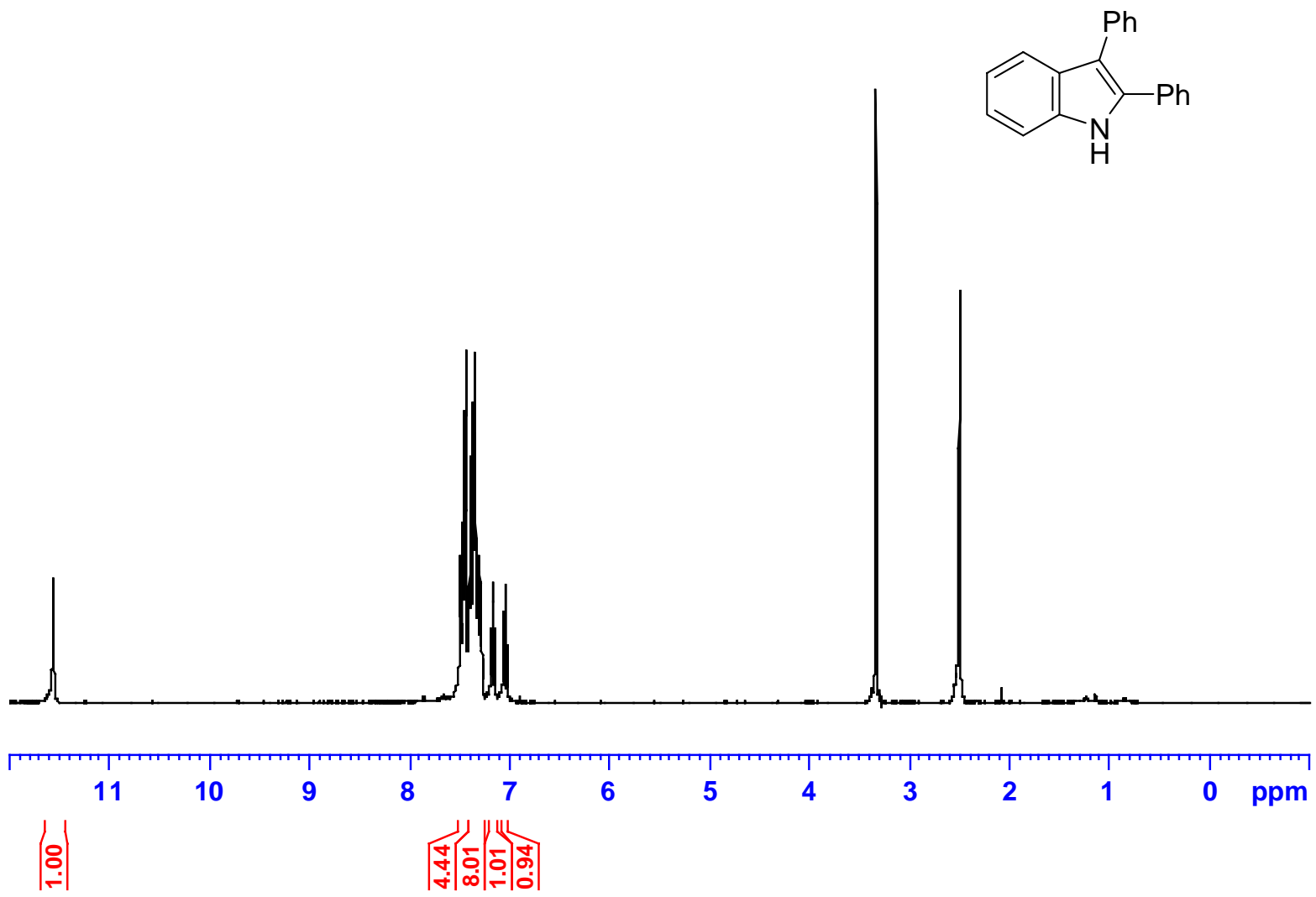


2,3-Diphenyl-1H-indole (3) $\left({ }^{13} \mathrm{C}\right.$ NMR) (Table 2, entry 1)

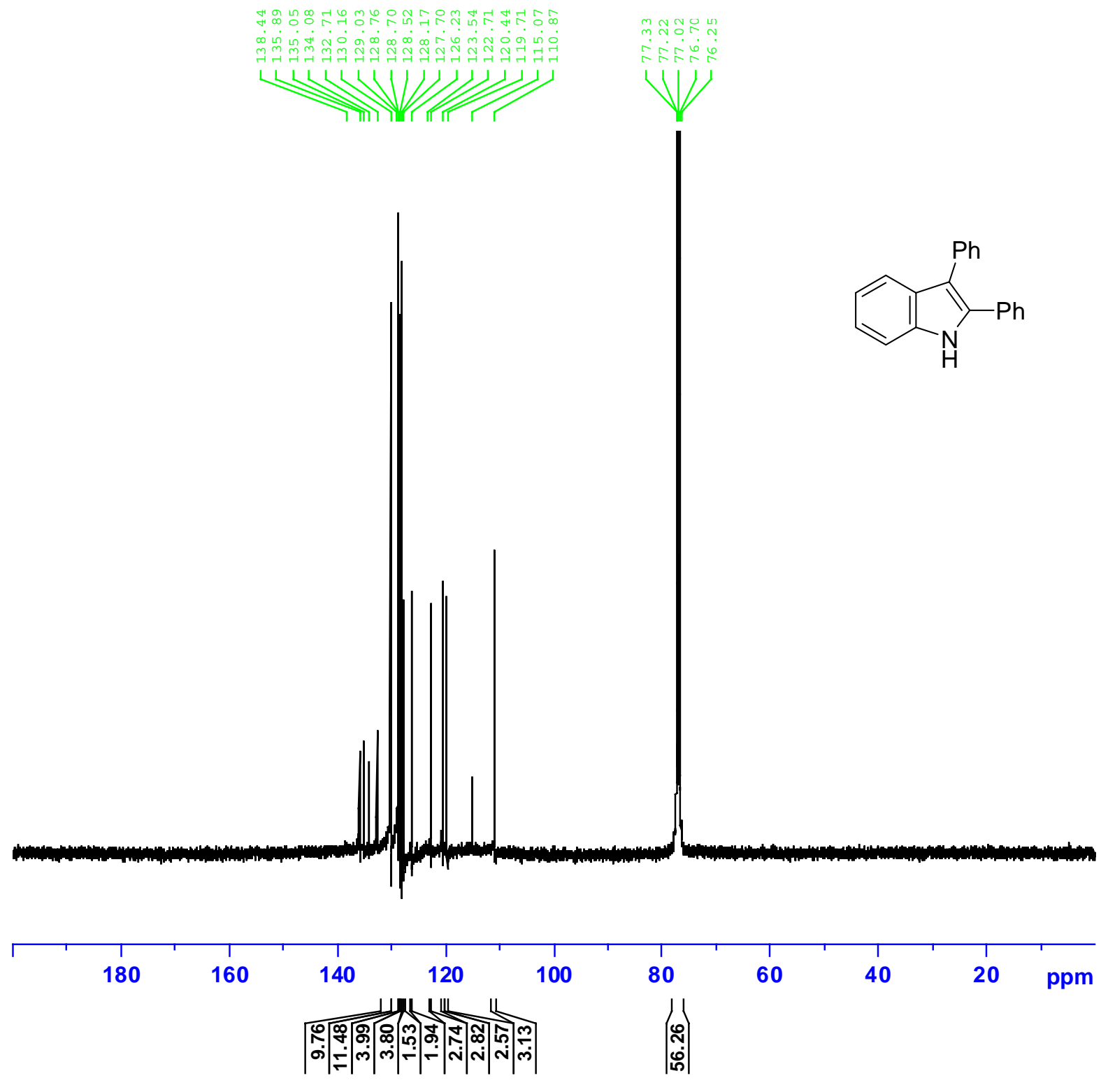


Methyl 6-(2,3-diphenyl-indolyl)carboxylate (8) $\left({ }^{1} \mathrm{H}\right.$ NMR.) (Table 2, enry 5):

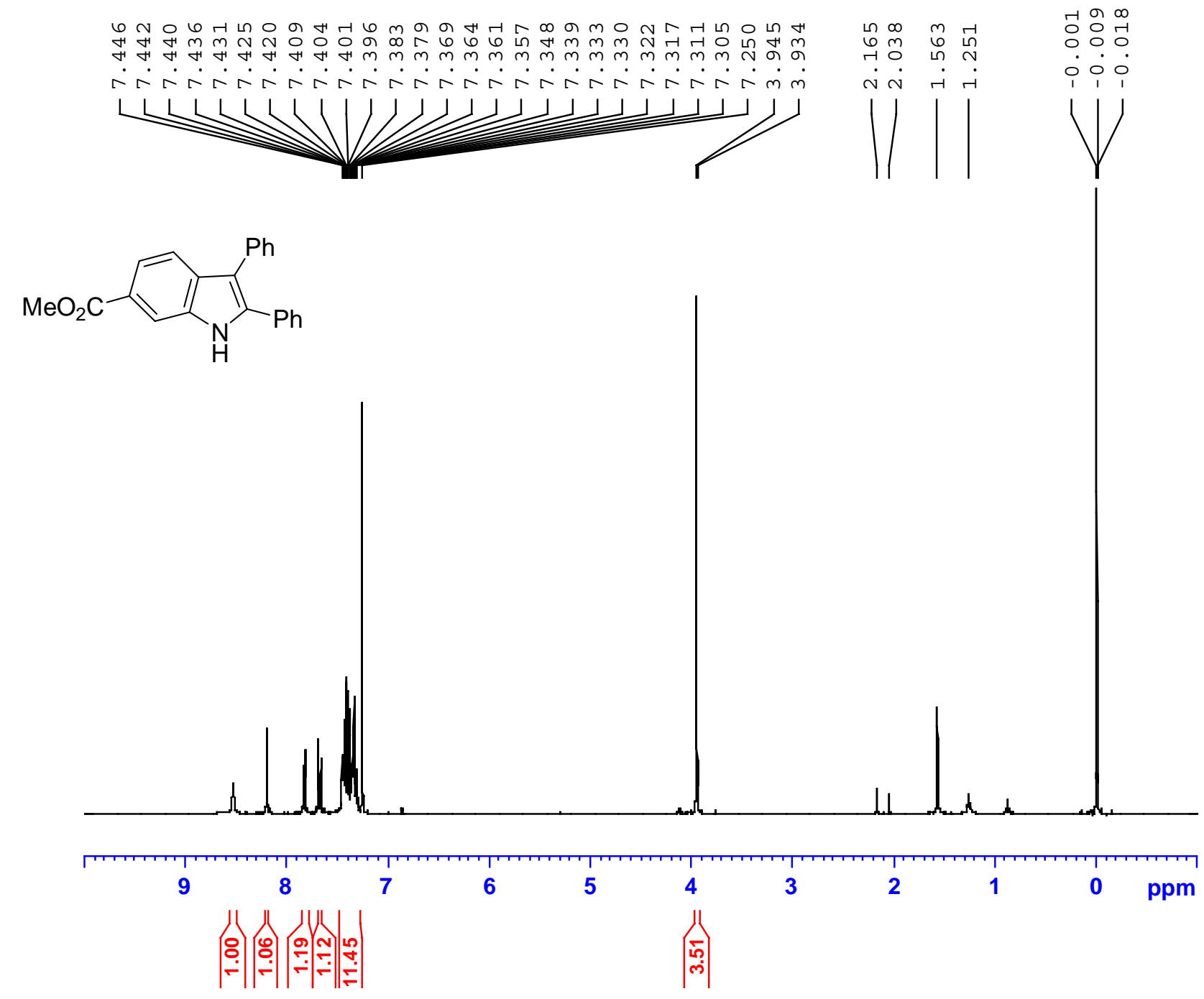


Methyl 6-(2,3-diphenyl-indolyl)carboxylate (8) $\left({ }^{13} \mathrm{C}\right.$ NMR.) (Table 2, enry 5):

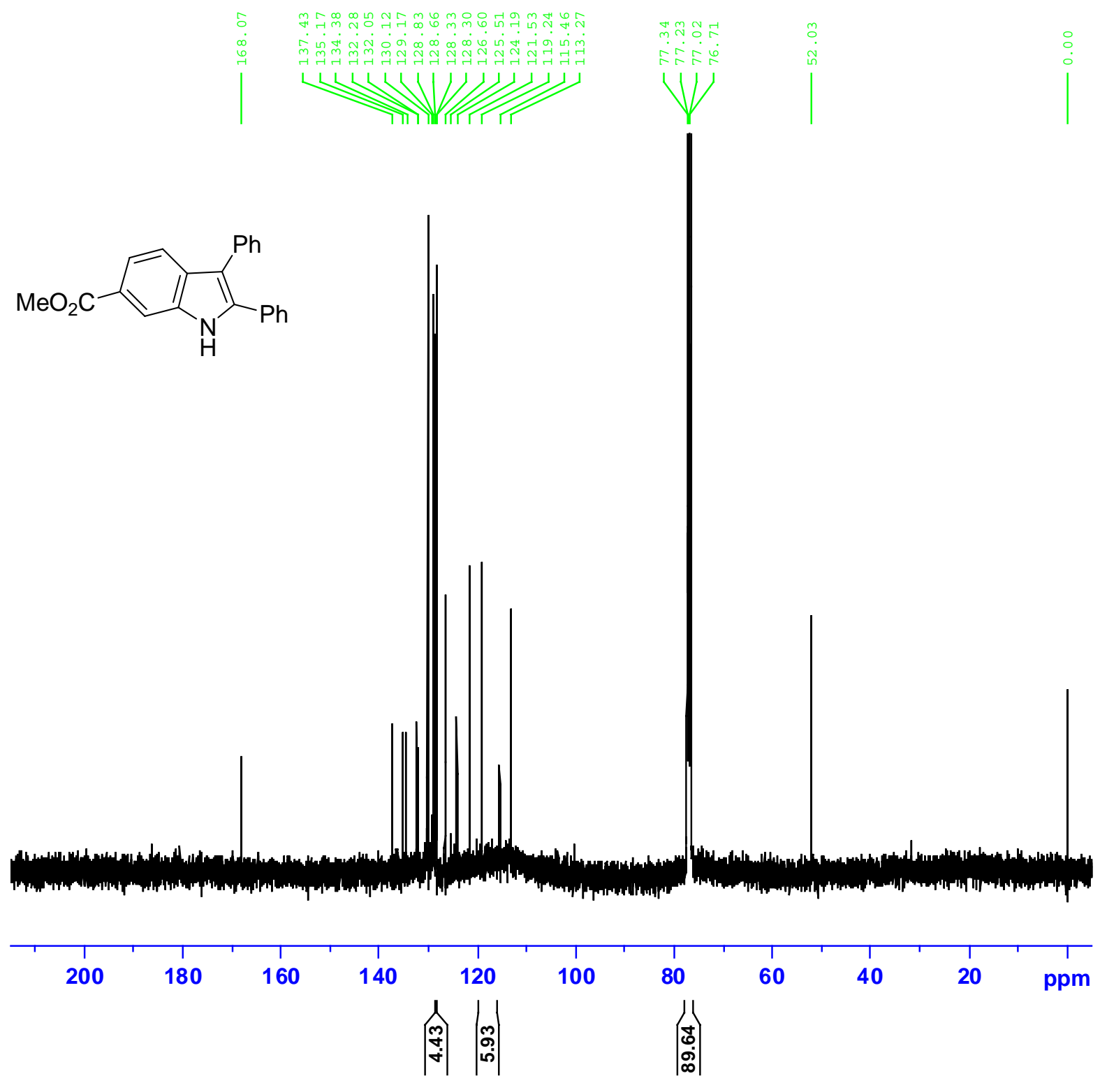


Methyl 6-[2-(p-methylphenyl)-3-phenylindolyl]carboxylate (7) $\left({ }^{1} \mathrm{H}\right.$ NMR) (Table 2 , entry 4):
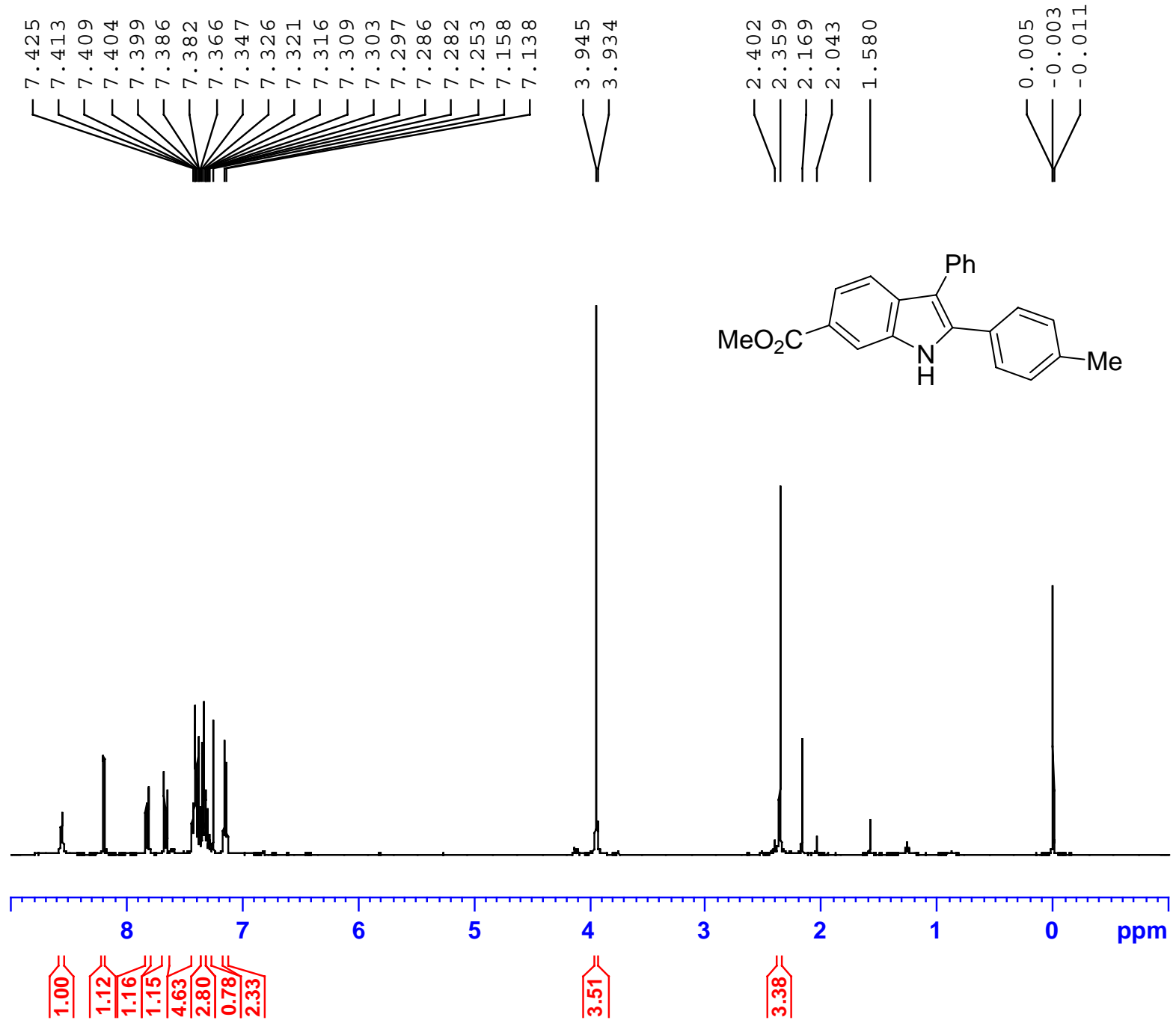
Methyl 6-[2-(p-methylphenyl)-3-phenylindolyl]carboxylate (7) $\left({ }^{13} \mathrm{C}\right.$ NMR) (Table 2, entry 4):

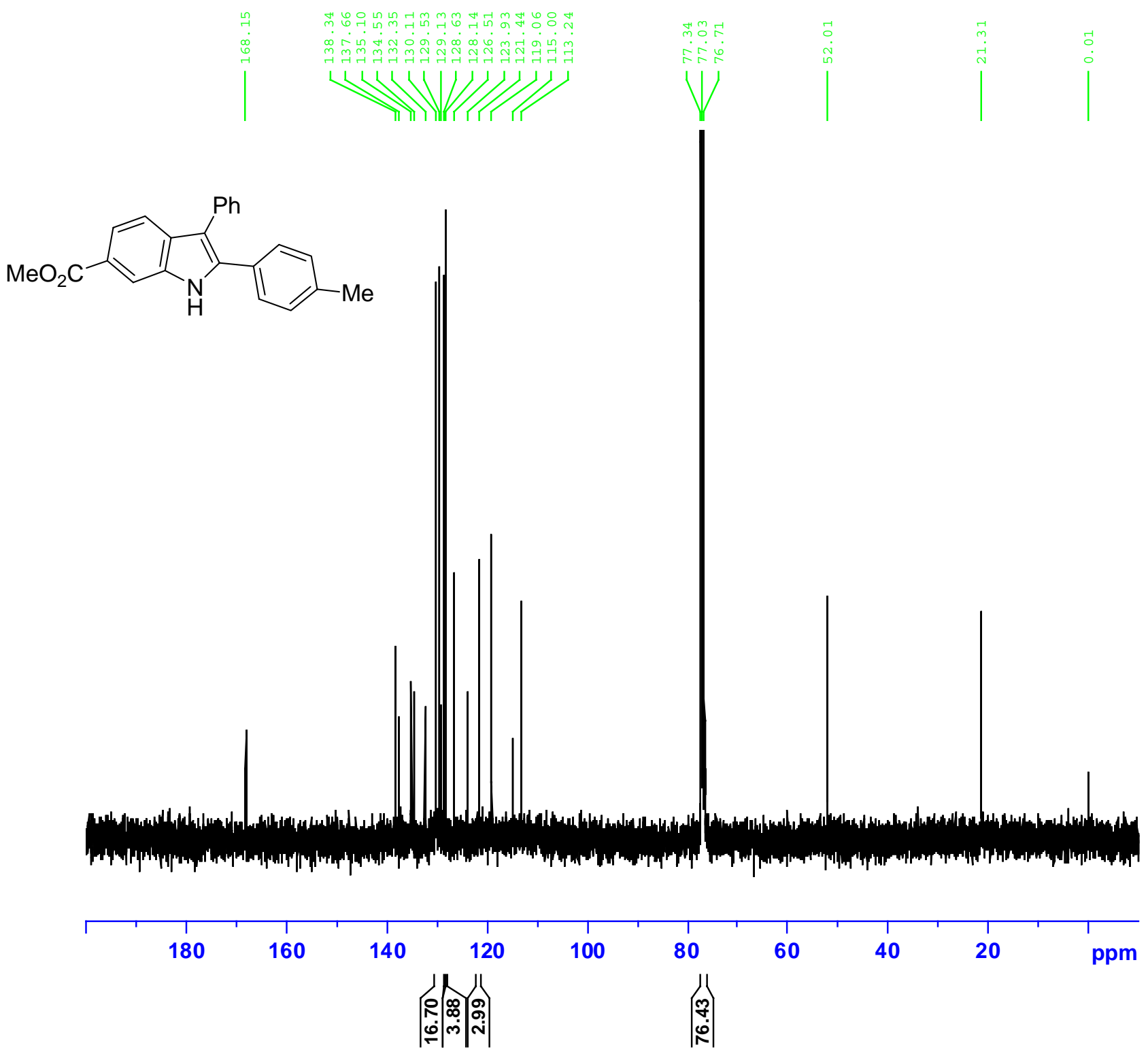


2,3-Diphenyl-5-cyano-1H-indole (11) ( ${ }^{1} \mathrm{H}$ NMR) (Table 2, entry 8):

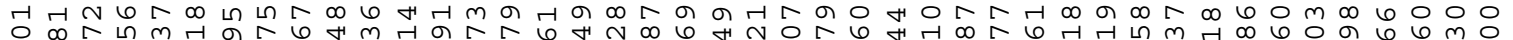

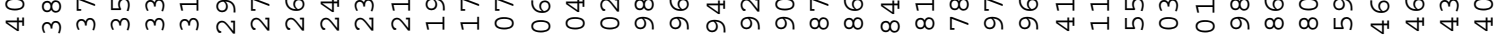

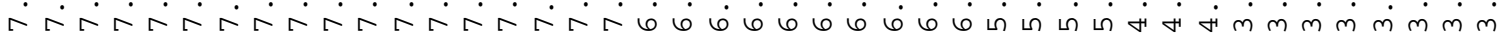

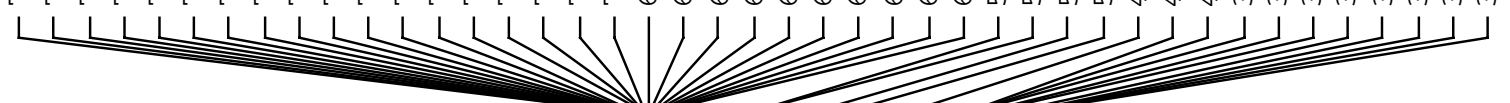<smiles>N#Cc1ccc2[nH]c(-c3ccccc3)c(-c3ccccc3)c2c1</smiles>

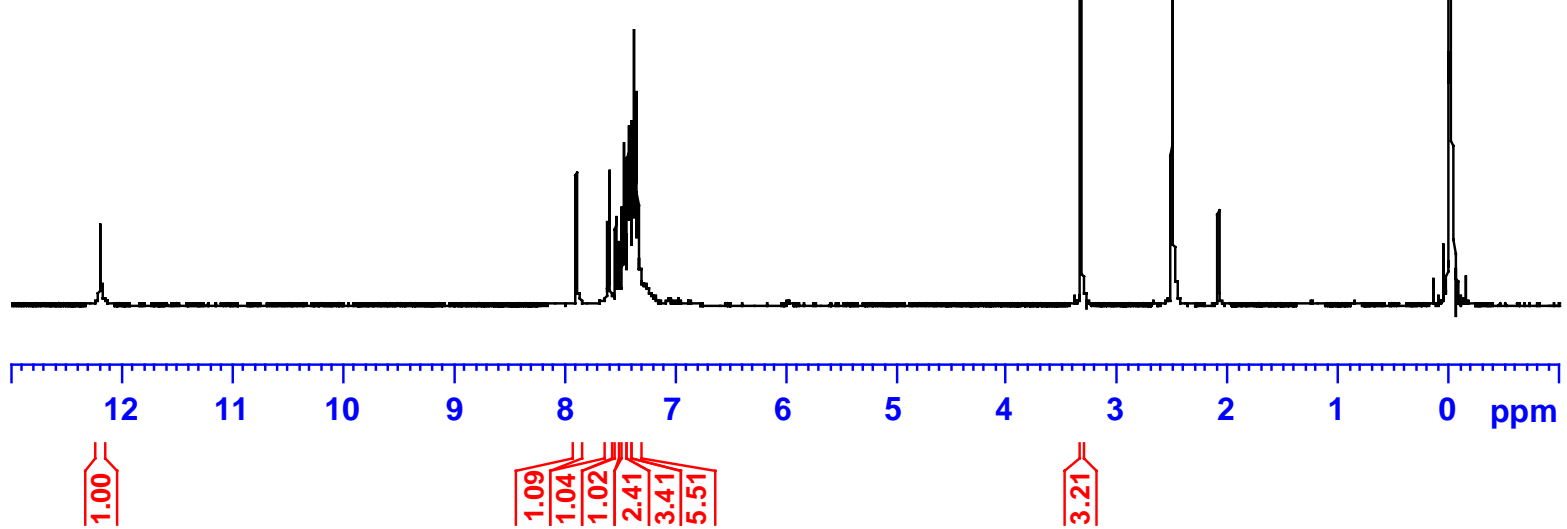


2,3-Diphenyl-5-cyano-1H -indole (11) $\left({ }^{13} \mathrm{C}\right.$ NMR) (Table 2, entry 8):

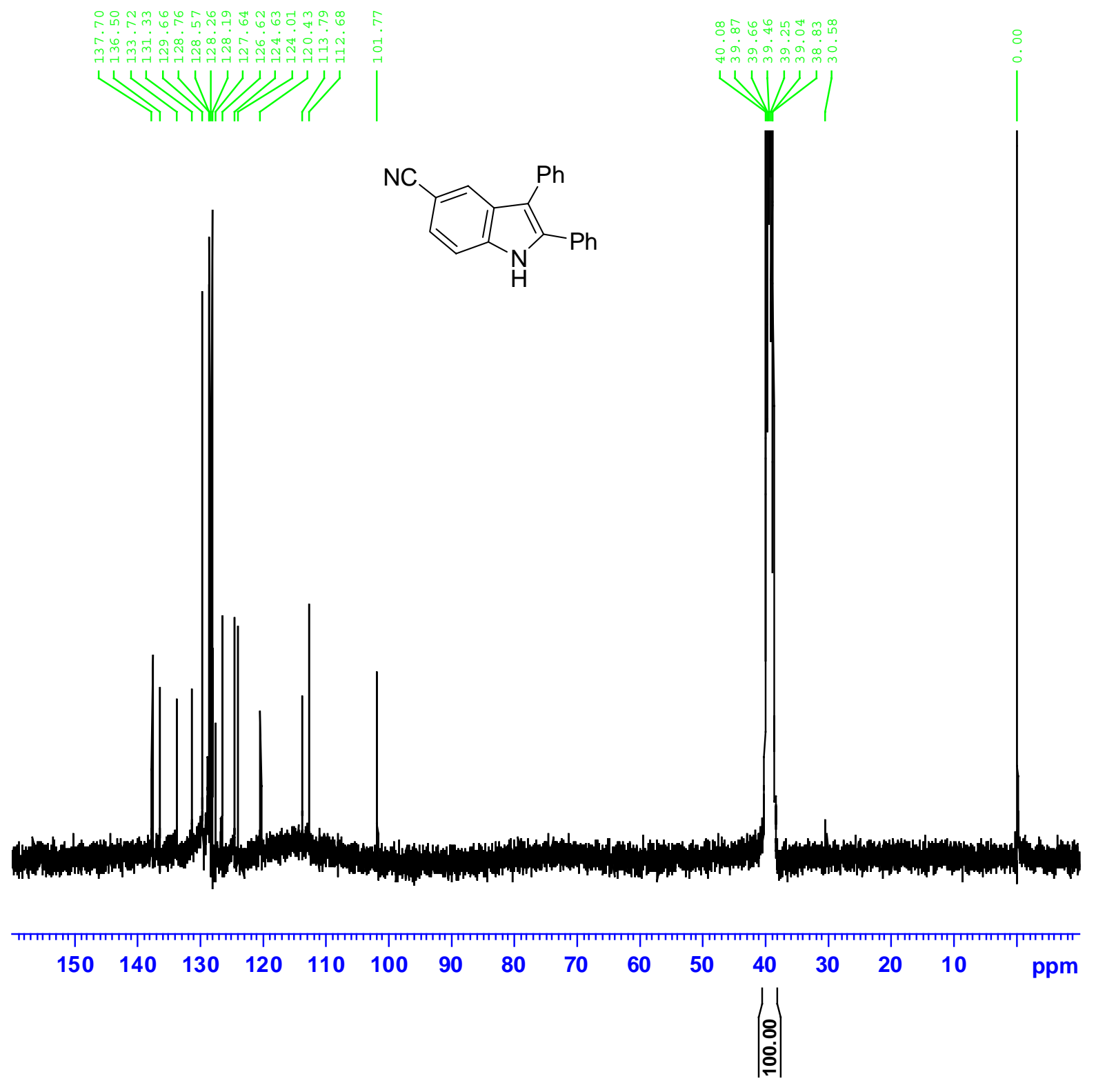


2-Phenyl-3-(p-methoxyphenyl)-1H-indole (5) ( ${ }^{1} \mathrm{H}$ NMR)(Table 2, entry 2):
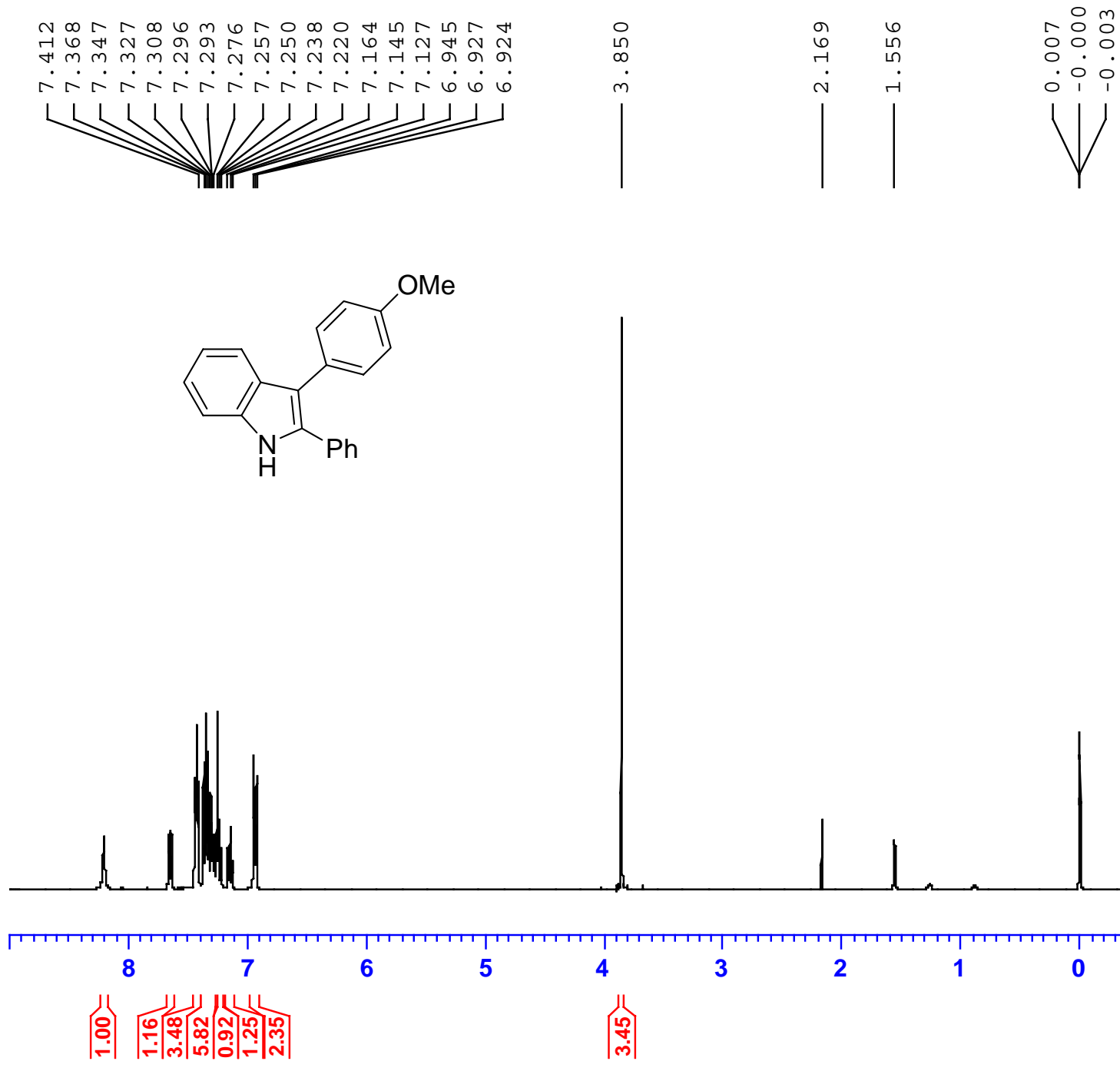
2-Phenyl-3-( $\boldsymbol{p}$-methoxyphenyl)-1H-indole (5) $\left({ }^{13} \mathrm{C}\right.$ NMR)(Table 2, entry 2):

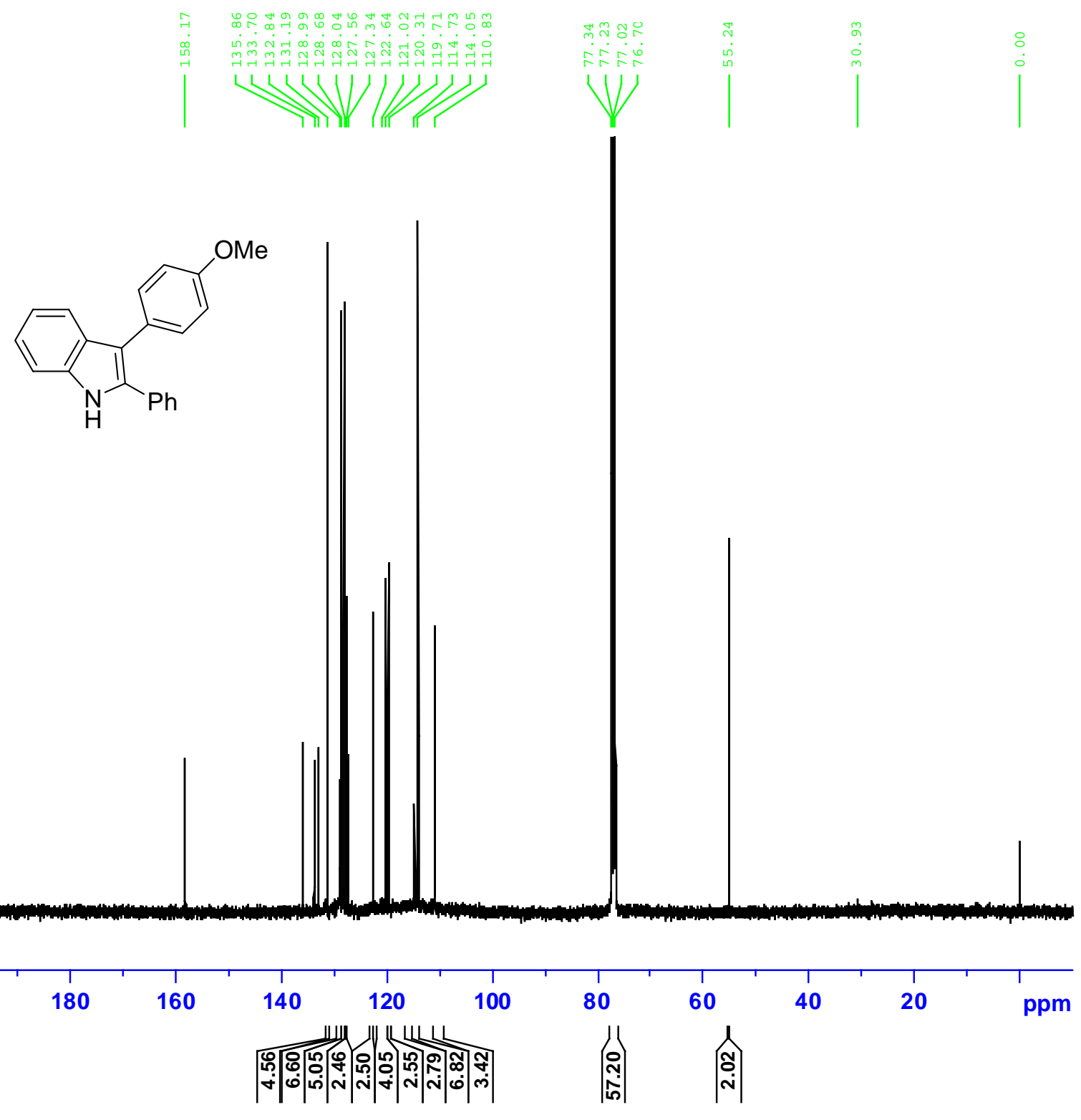


2-Phenyl-3-(p-methoxyphenyl)-5-cyano-1H-indole (12) $\left({ }^{1}\right.$ H NMR) (Table 2, entry 9):

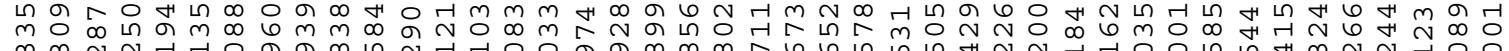

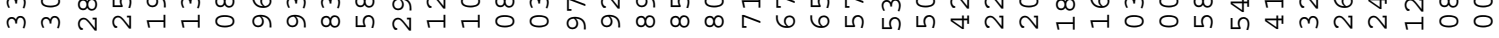
ararar
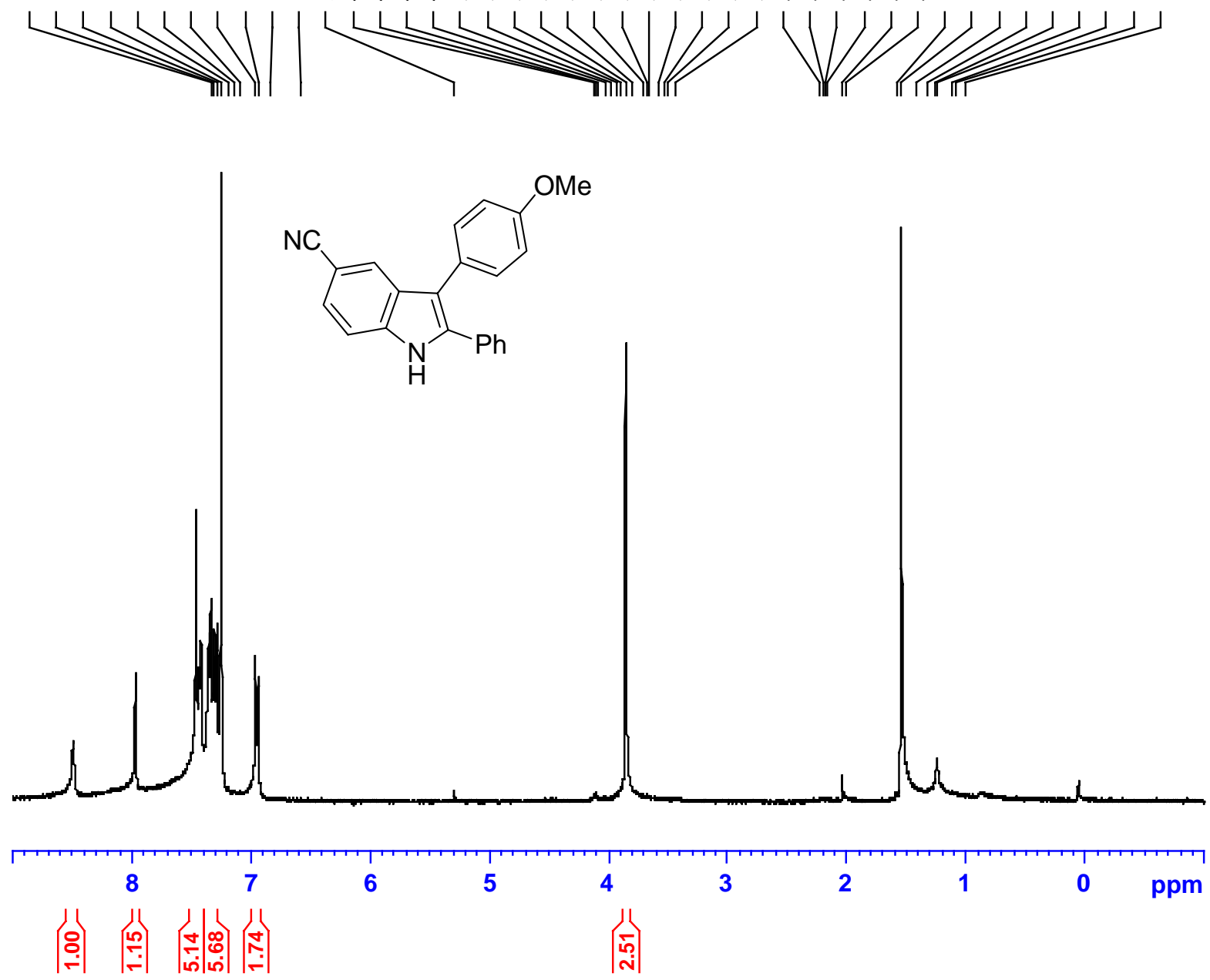
2-Phenyl-3-(p-methoxyphenyl)-5-cyano-1 $\boldsymbol{H}$-indole (12) $\left({ }^{13} \mathrm{C}\right.$ NMR) (Table 2, entry 9):

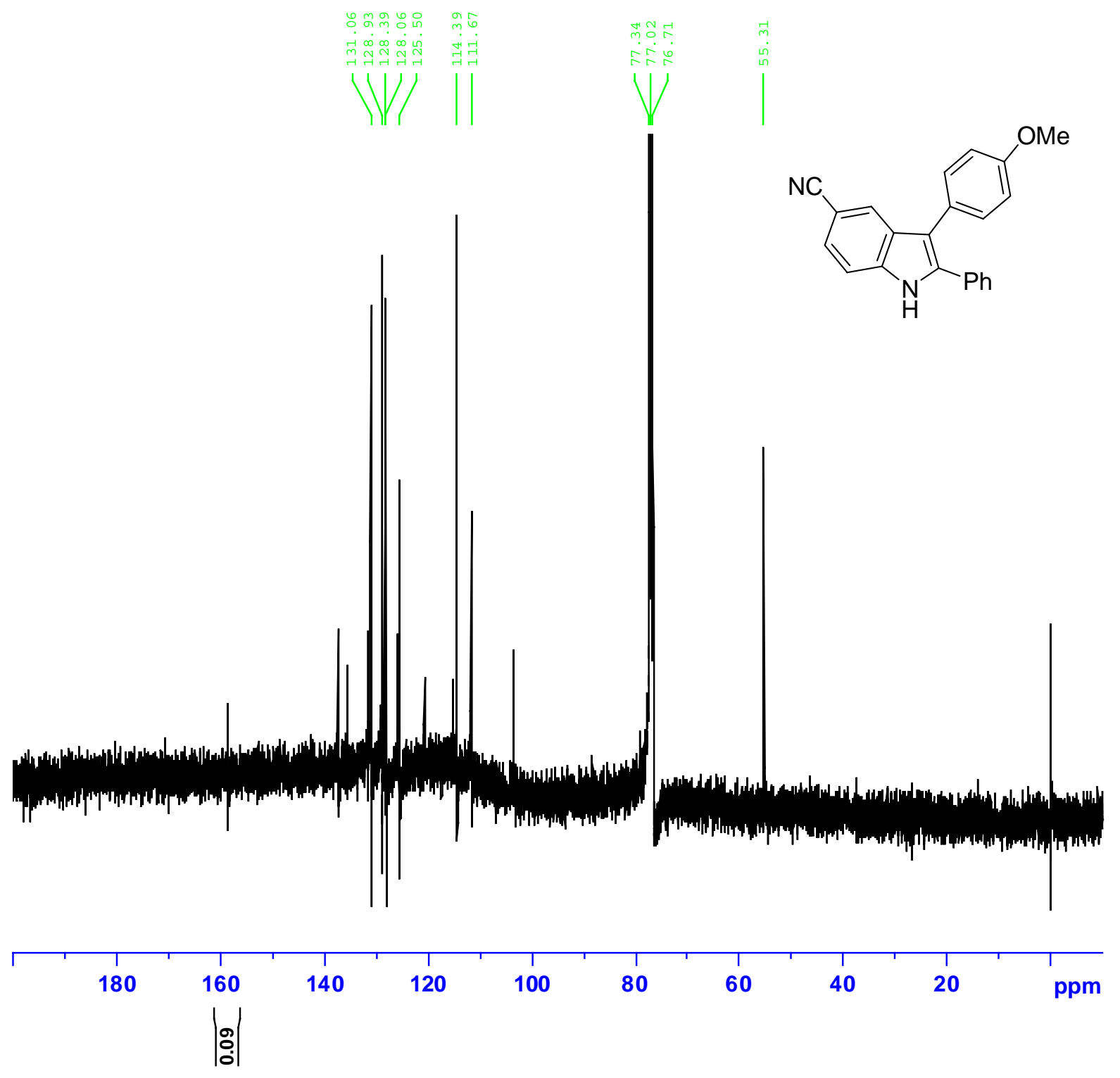


2-Phenyl-3-(p-methoxycarbonylphenyl)-1H-indole (6) ( ${ }^{1} \mathrm{H}$ NMR)) (Table 2, entry 3):

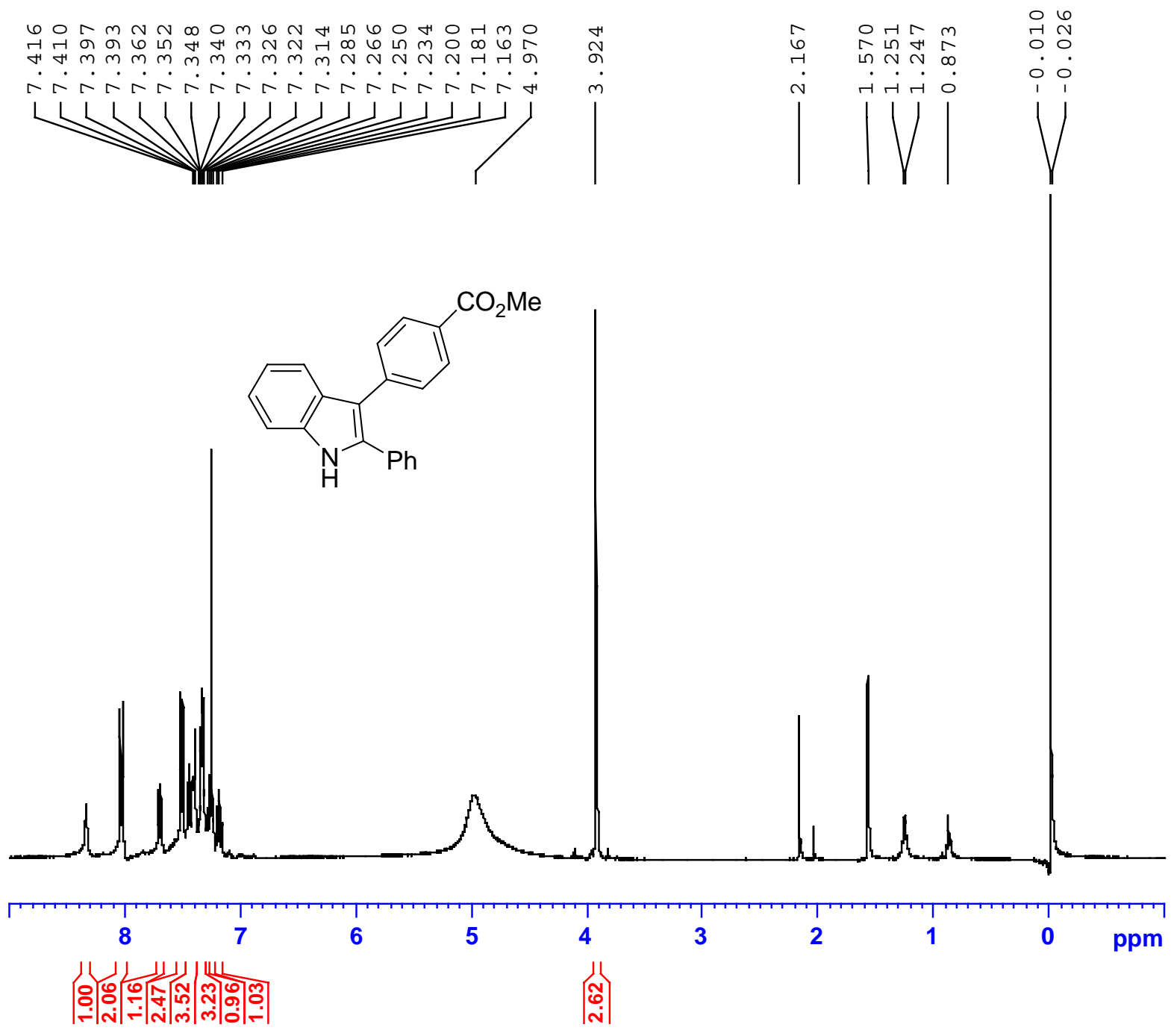


2-Phenyl-3-(p-methoxycarbonylphenyl)-1H-indole (6) $\left({ }^{13} \mathrm{C}\right.$ NMR)) (Table 2, entry 3):

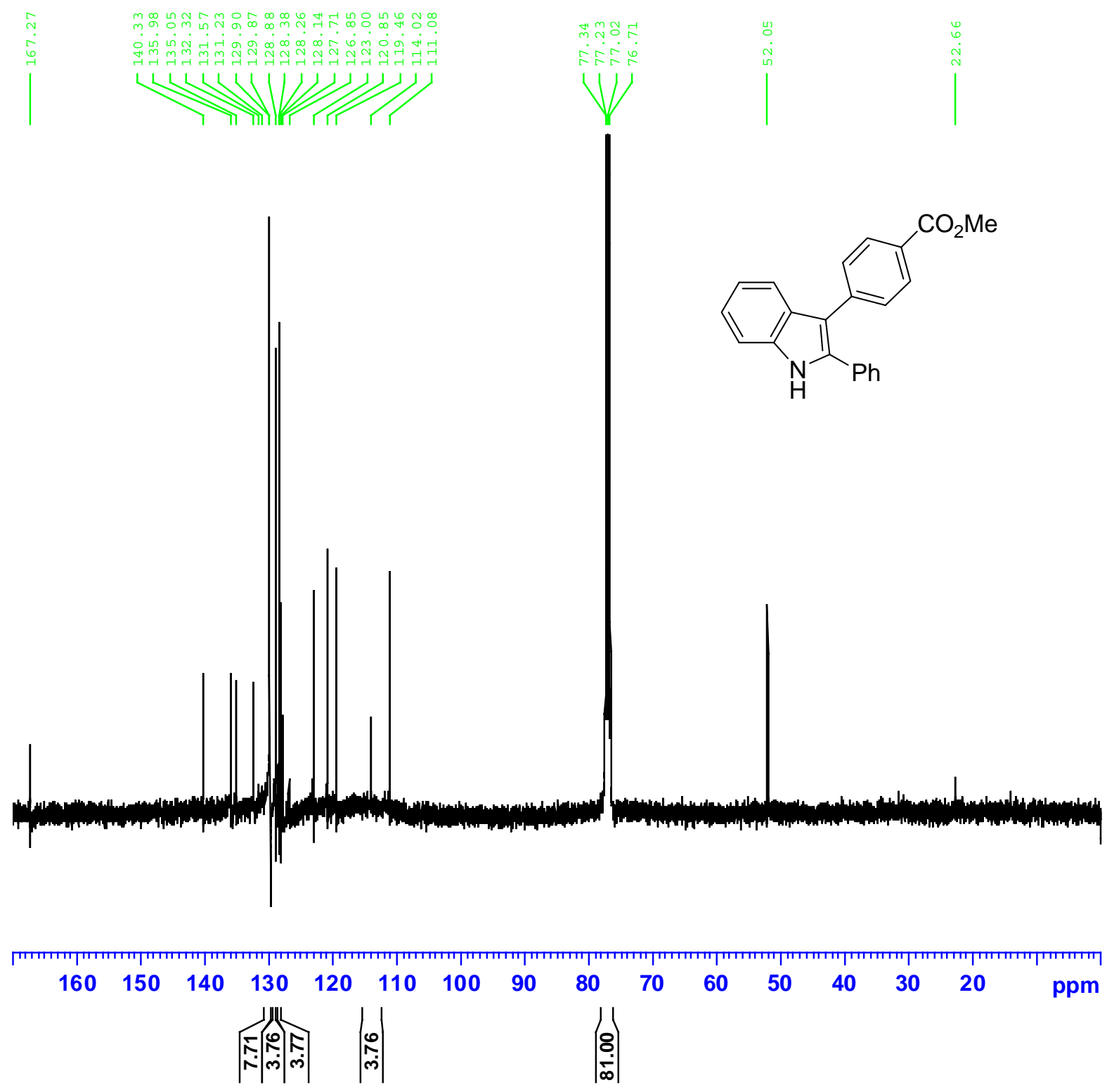


Methyl 6-[2-phenyl-3-(o-nitrophenyl)indolyl]carboxylate (9) ( ${ }^{1} \mathrm{H}$ NMR) (Table 2, entry 6):

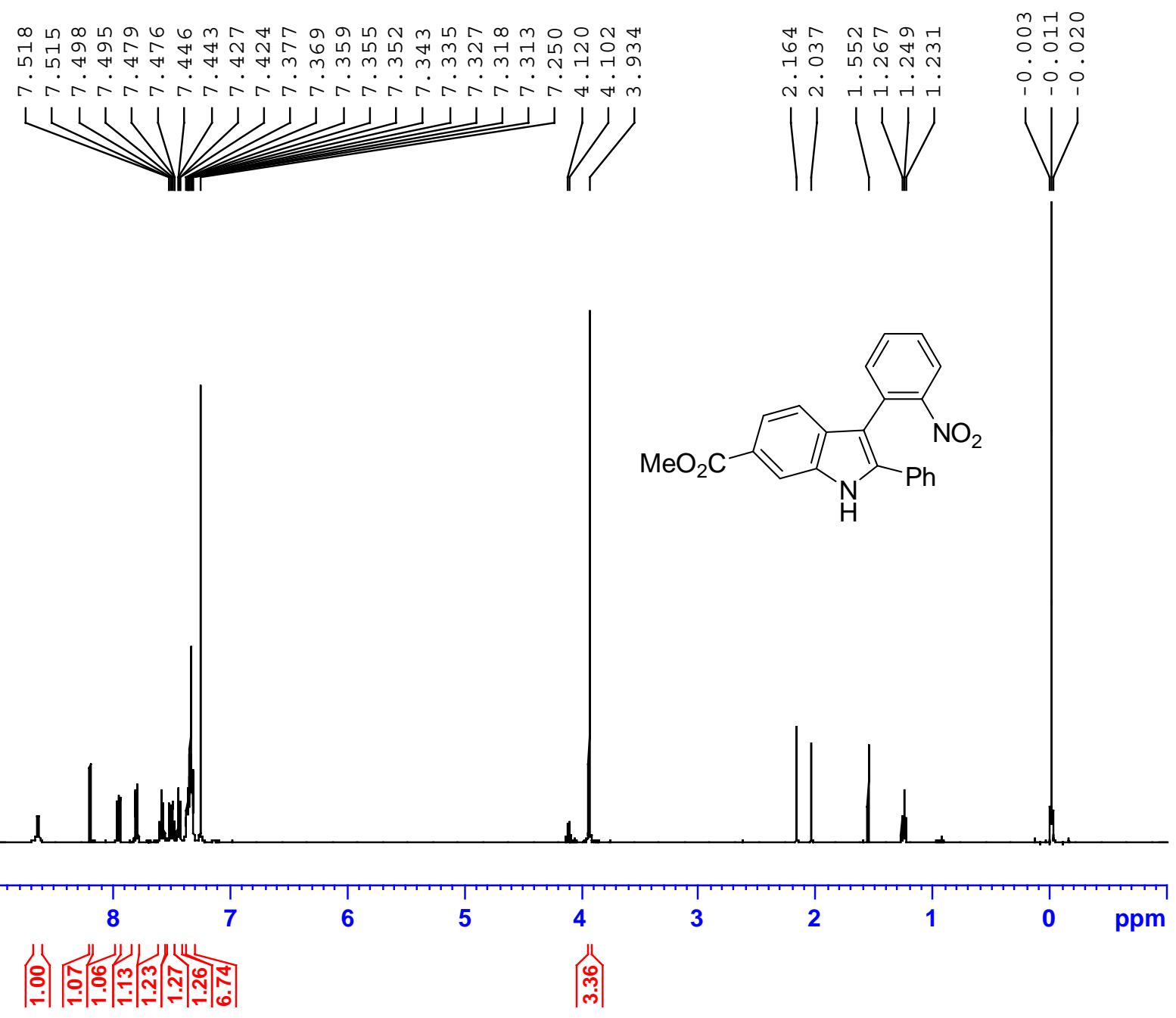


Methyl 6-[2-phenyl-3-(o-nitrophenyl)indolyl]carboxylate (9) $\left({ }^{13} \mathrm{C}\right.$ NMR) (Table 2, entry 6):

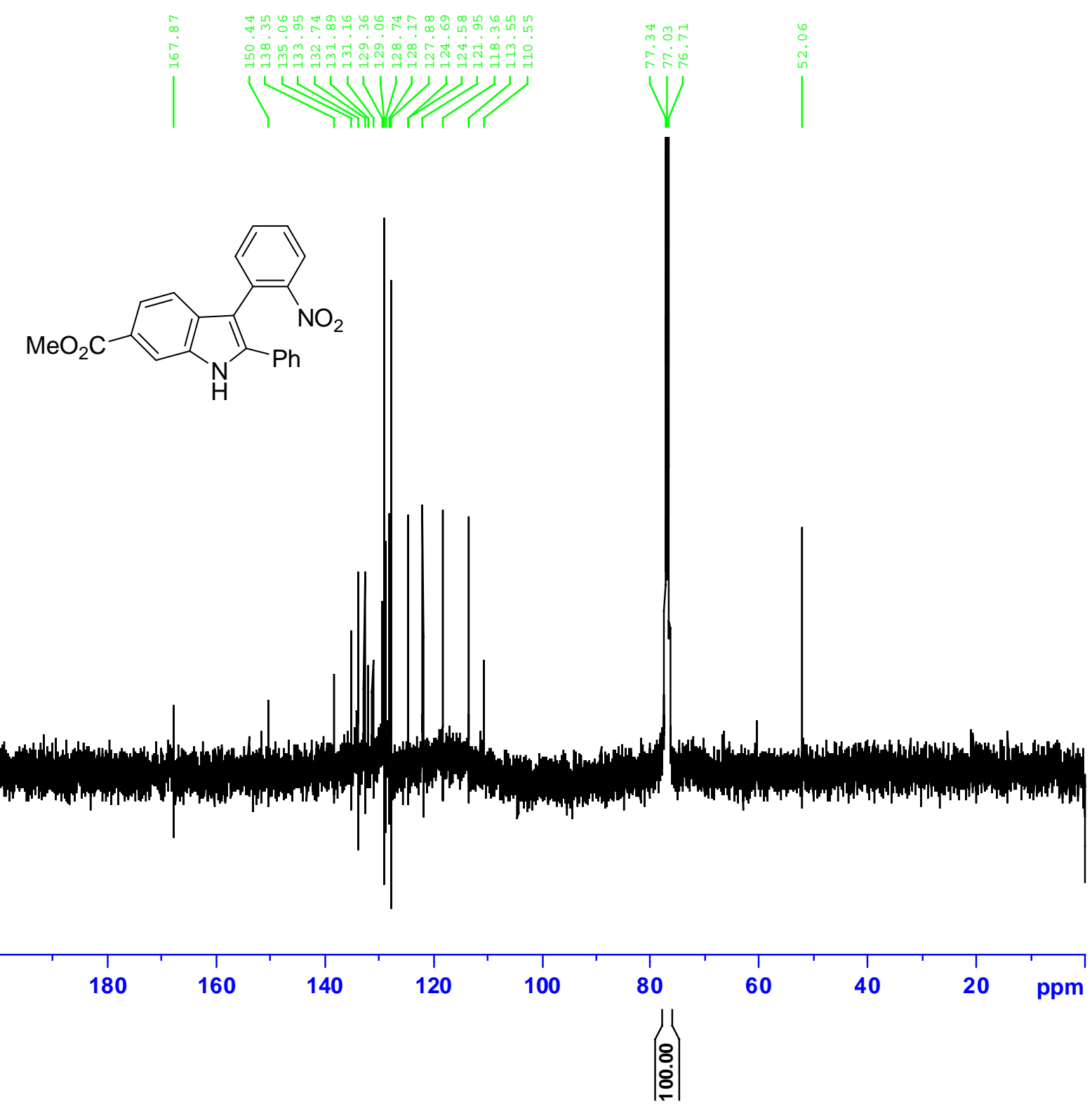




\section{2-Phenyl-3-(p-nitrophenyl)-1H-indole (10) $\left({ }^{1} \mathrm{H}\right.$ NMR) (Table 2, entry 7):}

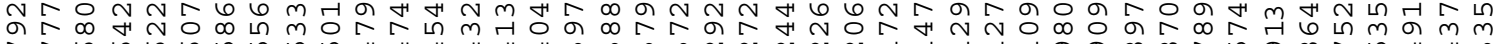
$\wedge \wedge 6666$ ถ

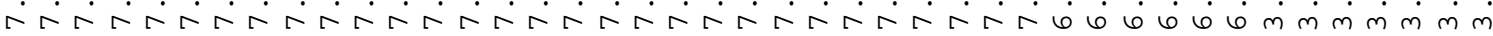

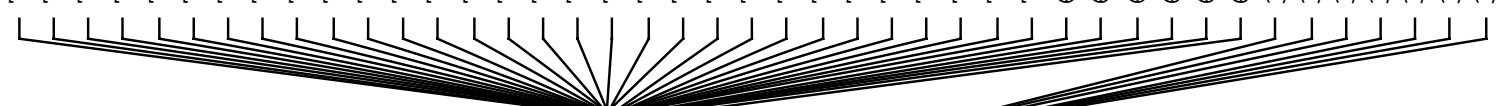<smiles>O=[N+]([O-])c1ccc(-c2c(-c3ccccc3)[nH]c3ccccc23)cc1</smiles>

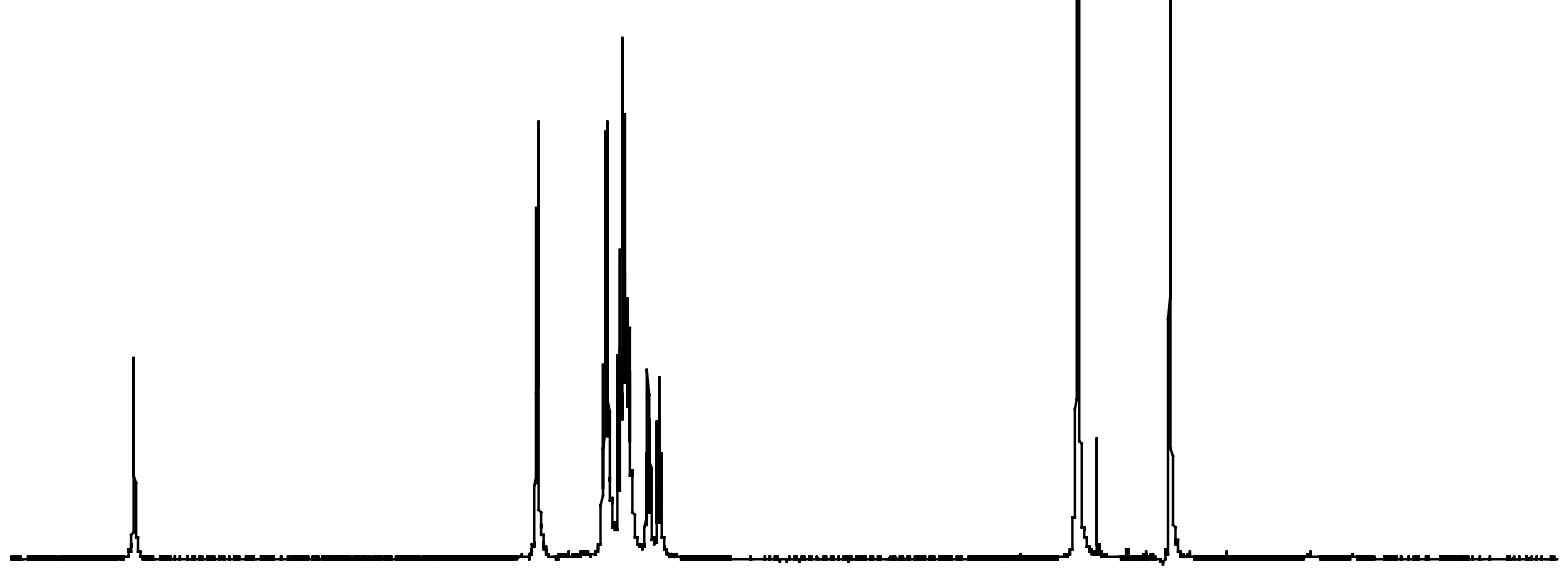

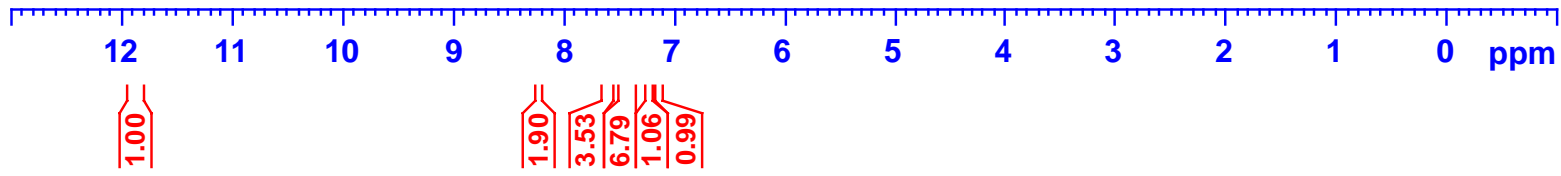


2-Phenyl-3-(p-nitrophenyl)-1H-indole (10) $\left({ }^{13} \mathrm{C}\right.$ NMR) (Table 2, entry 7):

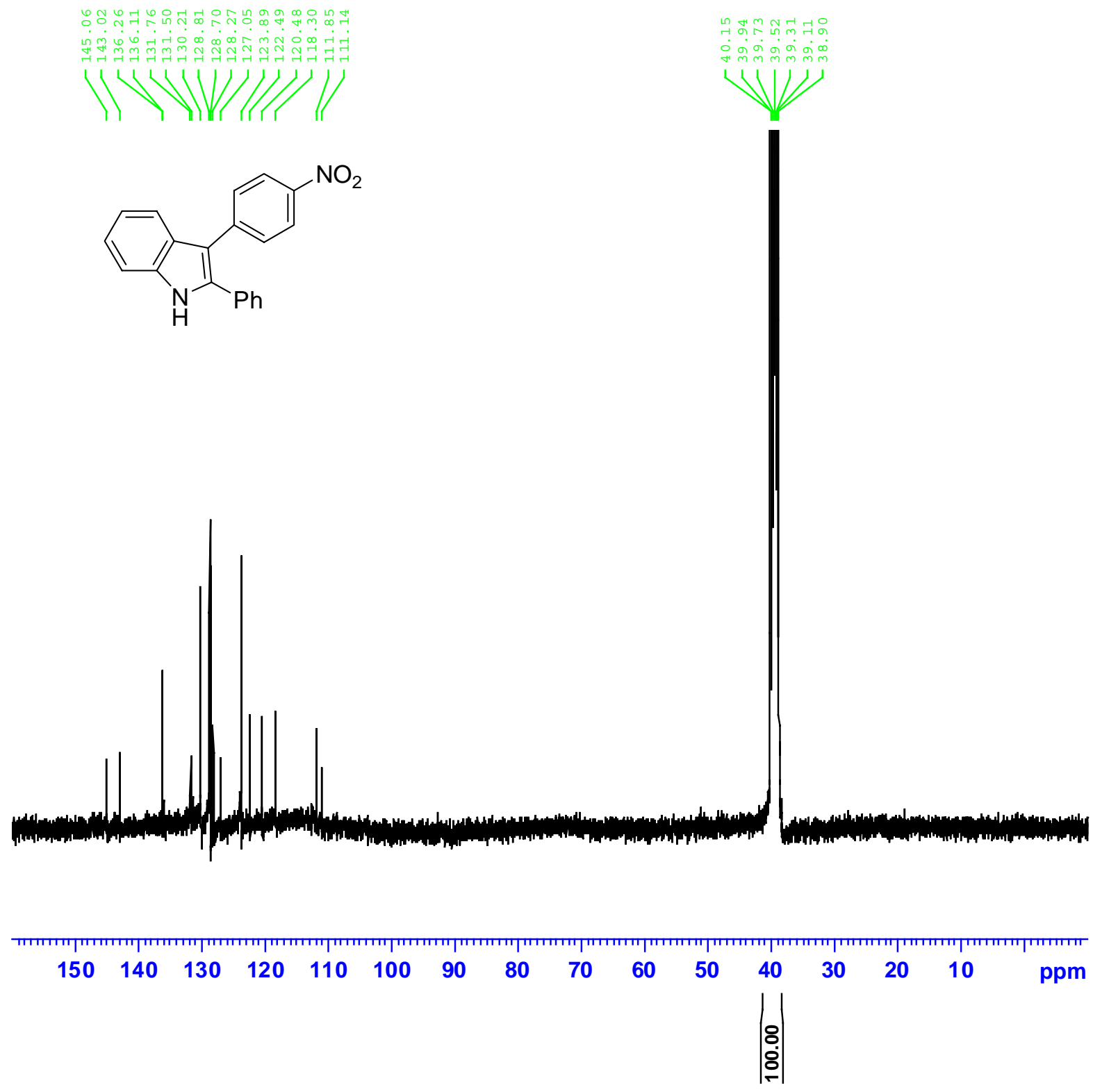


2-Phenyl-3-(o-nitrophenyl)-5-cyano-1H-indole (13) $\left({ }^{1} \mathrm{H}\right.$ NMR)(Table 2, entry 10):

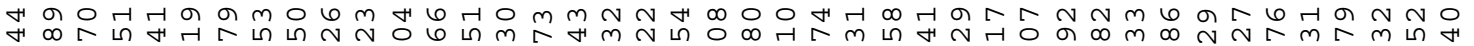
$\therefore 66666$ ம

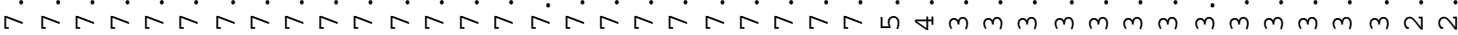

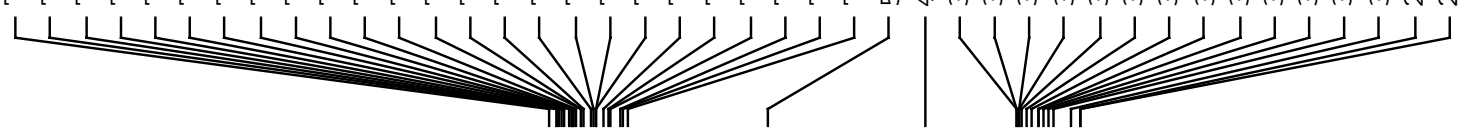<smiles>N#Cc1ccc2[nH]c(-c3ccccc3)c(-c3ccccc3[N+](=O)[O-])c2c1</smiles>

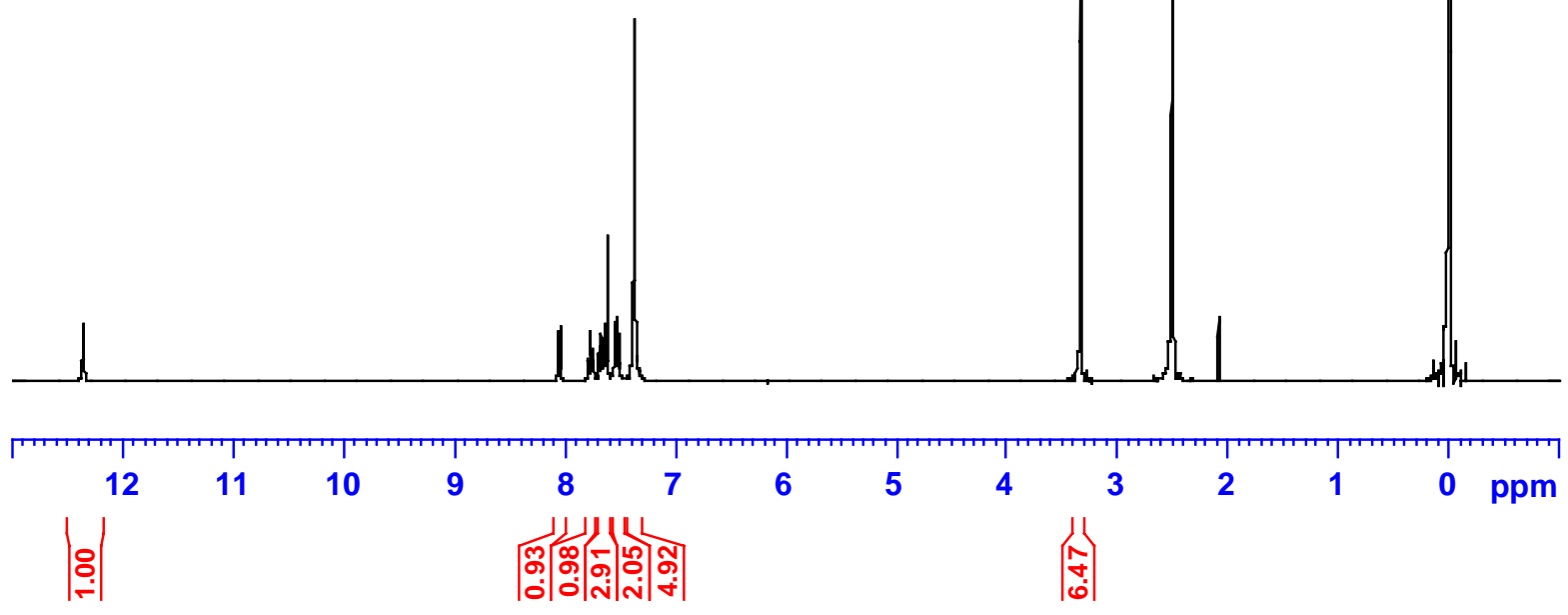


2-Phenyl-3-(o-nitrophenyl)-5-cyano-1H -indole (13) $\left({ }^{13} \mathrm{C}\right.$ NMR)(Table 2, entry 10):

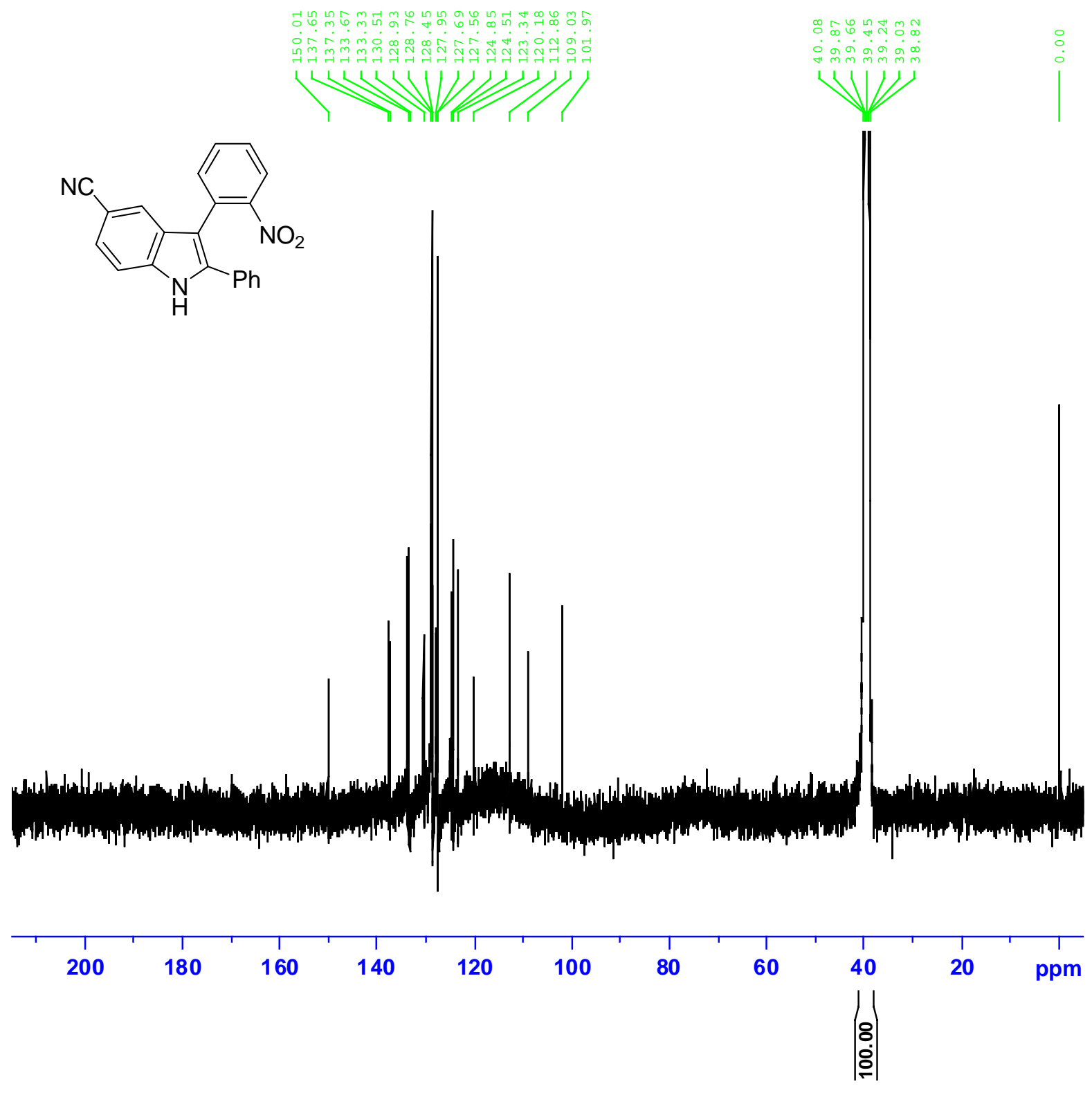

